\title{
Numerical Simulation of Fluid-Structure Interaction of D-shape Iced Conductor
}

\author{
You $\mathrm{Yi}^{1,2}$, Yan Zhitao ${ }^{1,3}$, Nie Xiaochun ${ }^{1, *}$, Yang Xiaogang ${ }^{1}$, \\ Li Wensheng $^{2}$, He Cheng ${ }^{2}$ and Liu Jun ${ }^{1}$ \\ ${ }^{1}$ School of Civil Engineering, Chongqing University, Chongqing 400045, China \\ ${ }^{2}$ Electric Power Research Institute, State Grid Xinjiang Electric Power Company, \\ Urumqi 830000, China \\ ${ }^{3}$ School of Civil Engineering, Chongqing University of Science \& Technology, \\ Chongqing 401331, China \\ E-mail: nxc@cqu.edu.cn \\ ${ }^{*}$ Corresponding Author
}

Received 28 March 2019; Accepted 24 July 2019;

Publication 27 August 2019

\begin{abstract}
At present, the numerical simulation on the aerodynamic response and force of the iced conductor are mainly based on the quasi steady criterion, which ignored the interaction between the conductor and the flow field. This paper presents a numerical study of three kinds of fluid-structure interaction models for D-shape conductor. The effects of reduced velocity, degree of freedom and wind attack angle on aerodynamic response of the iced conductor are discussed. The results show that the rotational freedom has certain influence on the across-wind vibration. The mean value of drag coefficient decreases with the increase of wind attack angle, while the lift and moment coefficient increase with the increase of wind attack angle. When the maximum amplitude of vibration displacement occurs, the corresponding reduced velocity is not entirely consistent with that of the maximum aerodynamic force.
\end{abstract}

Keywords: Iced conductor, fluid-structure interaction, aerodynamic characteristics, lock-in.

European Journal of Computational Mechanics, Vol. 28_3, 147-170.

doi: 10.13052/ejcm1958-5829.2832

(c) 2019 River Publishers 


\section{Introduction}

Fluid-structure interaction occurs in many engineering fields, for example, ocean engineering, aerospace engineering, transmission lines and so on. Fluidstructure interaction can cause complex vibration of the structure, which may lead to the structural damage under certain unfavorable conditions.

Vortex-induced vibration is a common phenomenon of fluid-structure interaction, which caused by vortex shedding from structure. In early stage, wind tunnel test was the main method to study this kind of problem. A series of wind tunnel tests were carried out to investigate the vortex-induced vibration phenomenon (Gabbai and Benaroya, 2005; Griffin and Koopmarm, 1977, 1980; Sarpkaya, 2004; Williamson and Govardhan, 2004, 2008), which indicated that the vortex-induced vibration is a kind of limiting vibration. Khalak and Williamson (1996) investigated the vortex induced vibration of a cylinder with low mass damping ratio. It revealed that the response of the cylinder will bifurcate with the variation of reduced velocity, a lower branch and an initial branch, and the two branches are discontinuous. Moreover, three branches will appear, which corresponding to the initial, lower and upper branch, respectively, for the case of low mass damping ratio (Govardhan and Williamson, 2000).

Due to the high cost and long period of experiment, the Computational Fluid Dynamics (CFD) technology has received growing attention in recent years. Yang et al. (2008) investigated the dynamics of an elastic cylinder in a free stream, and found that a strong coupling scheme could have more stable computation than a weak coupling one when the solid object has less density than the surrounding fluid. The Reynolds-averaged Navier-Stokes (RANS) code equipped with the SST k-w turbulence model was applied for the simulation of vortex-induced vibration with low mass-damping under different reduced velocities by Pan et al. (2007), and the vortex shedding process was compared with the experimental results. The discrepancies between the numerical results and the experimental data in the upper branch region have been discussed. Kamakoti and Shyy (2004) developed an efficient interface to couple the fluid and structure by a bilinear interpolation technique to map loads from CFD surface mesh to a temporary structure mesh and then extracted the load vector on the beam element. Al-Jamal and Dalton (2004) used the Large Eddy Simulation (LES) method to calculate the vortex-induced vibration of the two-dimensional circular cylinder with the Reynolds number 8000, and the influence of mass ratio $\mathrm{M}^{*}$ and structural damping $\zeta$ on lock-in range of frequency were discussed in detail. Guilmineau and Queutey (2004) also 
investigated the dynamics and fluid forcing on an elastically mounted rigid cylinder with the SST k-w turbulence model for the case of low mass-damping. Wang and Zhou (2005) investigated the vortex induced vibration of square cylinder, and pointed out that the response of the square cylinder is dominated by the third order modes, which is different from that of the circular cylinder. Arun (2013) carried out a Direct Numerical Simulation (DNS) of flow past a finite length square cylinder at a Reynolds number of 250. In order to analyse the effect of aspect ratio on the flow field and associated integral parameters, the aspect ratio for 2, 3, 4 and 5 are discussed respectively. It indicated that the non-dimensional frequency, Strouhal number and drag coefficient increase with the increase of aspect ratio.

The above research objects of vortex-induced vibration mainly focused on the circular and square cylinder. D-shape iced conductor is one of the most common shapes of iced conductor in transmission lines. Since the aerodynamic coefficients of iced conductors are the basis for the study on the windinduced vibration of conductors, scholars have carried out lots of fundamental researches on the aerodynamic characteristics of iced conductors (Chabart and Lilien, 1998; Dyke and Laneville, 2008; Gurung et al., 2002; Shimizu and Ishihara, 2004). These researches mainly focused on the influence of the ice shape, wind velocity, ice thickness and wind attack angle on aerodynamic characteristics. Based on the curved beam theory, a C-shape iced conductor finite element galloping model was proposed by Yan et al. (2013). Compared with the conventional cable model, the proposed model presents higher accuracy in simulating the mechanical characteristics of iced conductor. Borna (2014) has done a lot of research work on prediction of galloping of transmission line. Borna et al. (2011) investigated the fluid-structure interaction of the threedegree-of-freedom crescent-shaped iced conductor based on the unsteady Reynolds-Averaged Navier-Stokes equations and the Spalart-Allmaras oneequation turbulence model. The results are validated against experiments, and it can be a reference for the fluid-structure interaction analysis of other types of iced conductor, and then Borna et al. (2013) also analysed the effects of initial orientation of the ice on flow-induced instabilities of an iced conductor under different velocities by aeroelastic numerical approach. It revealed that the failure of the Den-Hartog's aerodynamic criterion to predict all potential instability zones and provide evidences that galloping instability is a velocity-dependent and damping-controlled. Li et al. (2013b) studied the variation law of aerodynamic coefficients of D-shaped and crescent-shaped iced conductor with wind velocity and ice thickness by ADINA software with the Spalart-Allmaras (SA) turbulence model. Lou et al. (2014) investigated 
the aerodynamic characteristics of crescent and D-shape bundled conductors by the wind tunnel test. The influence of icing thickness, initial ice accretion angle and sub-conductor on the aerodynamic properties were investigated, and the galloping possibility of bundled conductors is discussed based on the Den Hartog criterion. Similarly, Liu et al. (2015) studied the aerodynamic characteristics of the crescent iced single conductor by the numerical simulation, the results agree well with the test results, and then the Den Hartog and O. Nigol mechanism were used to predict the galloping of iced conductors. Ma et al. (2014) carried out experimental investigation and theoretical analysis on galloping of crescent-shaped and fan-shaped iced conductors. The influence of turbulence on aerodynamic coefficients and stability criterion was analyzed. Liu et al. (2013) investigated the hysteresis phenomenon in the galloping of the D-Shape iced conductor theoretically. The influence of the dynamic angle of attack, span length, initial tension, and conductor mass on the galloping were discussed. Recently, an isogeometric collocation method based on the nonuniform rational $\mathrm{B}$-splines was adopted to analyse the large amplitude, low frequency, and self-excited vibrations of a D-shaped iced conductor by Zhang et al. (2017). However, the focus of this paper is to propose an efficient numerical method, not to analyse the vortex-induced vibration of D-shape conductor.

Currently, most of the researches of fluid-structure interaction were focused on the circular and square cylinder. The relevant studies on fluid structure interaction of iced conductor were mainly focused on the theory of galloping, and the influence factors of aerodynamic coefficients. The numerical simulation of the aerodynamic response and force of the iced conductor were mainly based on the quasi steady criterion, which ignored the interaction between the conductor and the flow field. To the authors' knowledge, the research on fluid-structure interaction of iced conductor as a fully coupled problem is far from complete, especially the vortex-induced vibration. To fill the gaps, the weak-coupling method and SST k-w turbulence model are applied to deal with the interaction problem between the fluid and structure in this paper. Then the effects of reduced velocity, initial wind attack angle and degree of freedom on the aerodynamic response of the iced conductor are discussed.

\section{Numerical Method}

\subsection{Parameter Setting}

The cross section parameters of model are based on the classical D-shape iced conductor model given by Yu et al. (1995), where the diameter of the 


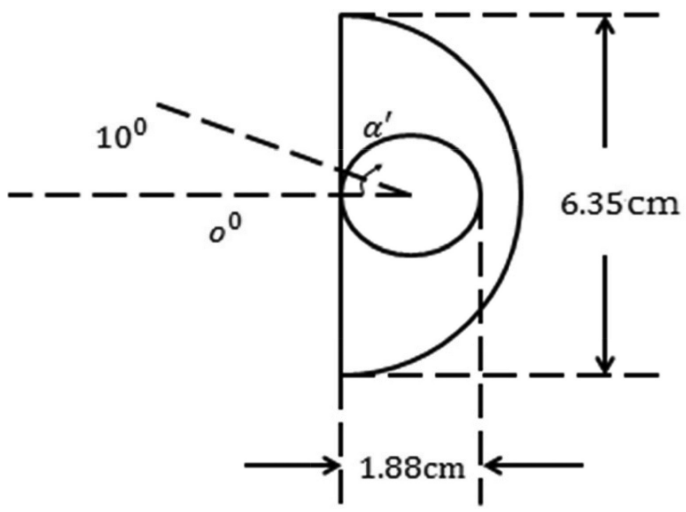

Figure 1 Schematic of the cross section for D-shape iced conductor.

conductor is $18.8 \mathrm{~mm}$, and the maximum size of iced conductor is $63.5 \mathrm{~mm}$. The schematic of the cross section for D-shape iced conductor are shown in Figure 1.

Numerical simulations are performed to treat the icing and conductor as a whole, regardless of the composition difference and interactions between them. The other parameters of the iced conductor are as follows: the mass ratio is 248 , which belongs to high mass ratio range, the natural frequency of the model in along-wind and across-wind is $4.0 \mathrm{~Hz}$, the damping ratio is 0.00103 , the wind velocity $V$ is $1.37 \sim 2.58 \mathrm{~m} / \mathrm{s}$, and the corresponding reduced velocity $V r(V r=V / f n * D, D$ is the maximum section size of iced conductor, $f n$ is the natural frequency of D-shape iced conductor in acrosswind) is from $5.421 \sim 10.149$, the range of Reynolds number is from 6000 to 11200 .

\subsection{Computational Domain Setting}

The calculated 2D model and grid are shown in Figure 2. The distance between the model and the left boundary is 25D. In order to ensure the full development of turbulence, the distance between the model and the right boundary is 35D. To meet the requirement of blocking rate, the distance between the model to the upper boundary and to the lower boundary is 30D. The fluid domain is discretized by structured grids. In order to make use of the dynamic and sliding grid to update the model grid, the fluid domain is divided into 8 regions (as shown in Figure 2(a)). The numerical simulation of the fluidstructure interaction is carried out by assigning different grid motion attributes 


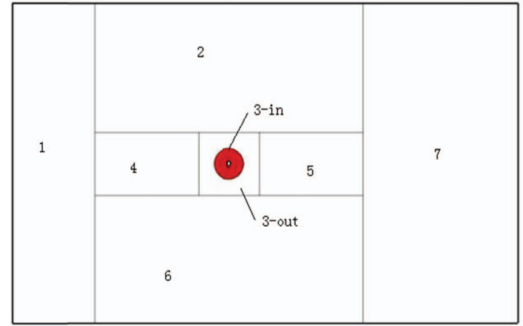

(a)

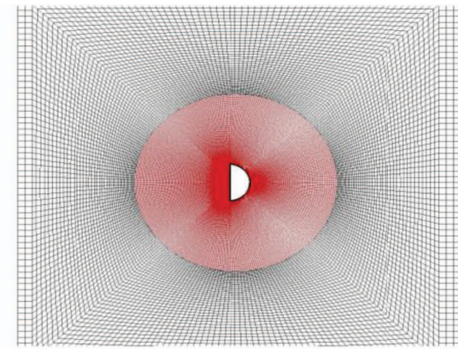

(b)

Figure 2 The numerical calculation model $\left(0^{\circ}\right.$ initial attack angle). (a) Computational domain and (b) Grid of near wall region.

to different regions. The grid region is updated by using the mixed mode of dynamic grid and sliding grid model. The data transfer and exchange between different fluid regions are realized by setting the interface. The grid around the model is rigidly synchronized with the motion of model by the dynamic grid macro commands. The flow runs from the left to the right, the left and right boundaries are the Velocity-inlet and Pressure-out respectively. The Opertating-pressure is set to 0 . The upper and lower boundaries are defined as symmetric boundary, and the No-slip wall is adopted on the model surface. Among them, the grid regions 1 and 7 are static regions, the grid regions 2 and 6 are the across-wind single-degree-of-freedom rigid body motion regions, the grid regions 3-in and 3-out are the multi-degree-of-freedom rigid body motion region, and the grid regions 4 and 5 are the along-wind single-degreeof-freedom sliding grid region. In addition, the grid refinement is performed to meet the requirements of turbulence model in the sensitive areas such as the near wall region. The mesh scale of the model surface is $0.00025 \mathrm{D}$ (Figure 2(b)).

\subsection{Computational Theory}

The weak-interaction method and SST k-w turbulence model are applied to deal with the coupling problem between the fluid and structure. The incompressible two-dimensional Reynolds-Averaged Navier-Stokes (RANS) equations are solved by the FLUENT Software within each time step. Then use the Compute-Force-And-Moment command to obtain the drag, lift and moment force of the structure. Here, the structure is considered as a massstiffness-damping system, the governing equations of the three degree of 
freedom model of the structure can be expressed as:

$$
\begin{aligned}
\ddot{X}+2 \xi \omega_{0} \dot{X}+\omega_{0}^{2} X & =F_{D}(t) / M \\
\ddot{Y}+2 \xi \omega_{0} \dot{Y}+\omega_{0}^{2} Y & =F_{L}(t) / M \\
\ddot{Z}+2 \xi \omega_{0} \dot{Z}+\omega_{0}^{2} Z & =F_{M}(t) / I_{z}
\end{aligned}
$$

Where, the $\xi$ and $\omega_{0}$ are the damping ratio and frequency respectively, $M$ is the equivalent mass on unit length, $I_{z}$ is the rotary inertia around $Z$ axis, the $X, Y$ and $Z$ are the along-wind, across-wind and torsional displacement. The dimensionless definition of aerodynamic coefficients is as follows:

$$
\begin{aligned}
F_{D} & =\frac{1}{2} \rho V^{2} D \cdot C_{D} \\
F_{L} & =\frac{1}{2} \rho V^{2} D \cdot C_{L} \\
F_{M} & =\frac{1}{2} \rho V^{2} D^{2} \cdot C_{M}
\end{aligned}
$$

Where, $\rho$ is the fluid density, $V$ is the uniform inflow velocity, $D$ is the maximum section size of structure, $F_{D}, F_{L}$ and $F_{M}$ are the drag, lift and moment force respectively.

By writing a user-defined function (UDF), the Newmark- $\beta$ algorithm is loaded into the main program to solve these governing equations. Here, the case of across-wind vibration (one-degree-of-freedom model) is taken as an example to solve the governing equation (Equation (2)). This method is also suitable for the calculation of two-degree-of-freedom model and three-degreeof-freedom model. By the means of Newmark- $\beta$ algorithm, the velocity and displacement of the structure at the $i$-th time step can be expressed as $(i \geq 1)$ :

$$
\begin{aligned}
& \dot{Y}_{i}=\dot{Y}_{i-1}+\frac{\Delta t}{2}\left(\ddot{Y}_{i-1}+\ddot{Y}_{i}\right) \\
& Y_{i}=Y_{i-1}+\Delta t \dot{Y}_{i-1}+\frac{\Delta t^{2}}{3} \ddot{Y}_{i-1}+\frac{\Delta t^{2}}{6} \ddot{Y}_{i}
\end{aligned}
$$

From Equations (7) and (8), the expressions for $\dot{Y}_{i}$ and $\ddot{Y}_{i}$ can be derived as:

$$
\begin{aligned}
& \dot{Y}_{i}=\frac{3}{\Delta t}\left(Y_{i}-Y_{i-1}\right)-2 \dot{Y}_{i-1}-\frac{\Delta t}{2} \ddot{Y}_{i-1} \\
& \ddot{Y}_{i}=\frac{6}{\Delta t^{2}}\left(Y_{i}-Y_{i-1}-\Delta t \dot{Y}_{i-1}-\frac{\Delta t^{2}}{3} \ddot{Y}_{i-1}\right)
\end{aligned}
$$


Considering Equations (9) and (10), we rewrite the governing Equations (2) of the system as:

$$
\begin{aligned}
\tilde{k} Y_{i} & =\tilde{F}_{L}(t) \\
\tilde{k} & =k+\frac{3 c}{\Delta t}+\frac{6 M}{\Delta t^{2}} \\
\tilde{F}_{L}\left(t_{i}\right)= & F_{L}\left(t_{i}\right)+M\left(\frac{6 Y_{i-1}}{\Delta t^{2}}+\frac{6 \dot{Y}_{i-1}}{\Delta t}+2 \ddot{Y}_{i-1}\right) \\
& +c\left(\frac{3 Y_{i-1}}{\Delta t}+2 \dot{Y}_{i-1}+\frac{\Delta t}{2} \ddot{Y}_{i-1}\right)
\end{aligned}
$$

Where, $\tilde{k}$ and $\tilde{F}_{L}\left(t_{i}\right)$ are the equivalent stiffness and equivalent load at time $t_{i}$, respectively. $k=M \omega_{0}^{2}, c=2 \xi \omega_{0}$.

Hence, the acceleration, velocity and displacement of time $t_{i}$ can be obtained from Equations (9), (10) and (11) respectively. The dimensionless parameter $t^{\prime}=t_{i} V / D$. Similarly, the displacement and velocity response of the structure can be obtained at each time step. The moving velocity of the structure is applied to the grid to update the grid by the dynamic and sliding grid model. When the calculation of fluid field of the current time step is convergent, the calculated results are carried out to be the initial value of the next time step, and then the above cycle is repeated until the calculation result is stable.

\section{Results and Discussion}

\subsection{Influence of One-Degree-of-Freedom on Vibration}

In order to verify the validity of the simulation method of D-shape iced conductor, the flow around circular cylinder and the fluid-structure interaction of circular cylinder is calculated by this method, and the simulation results are compared with other's research, which is shown in Figure 3.

Figure 3(a) and (b) show the mean value of drag coefficient verses Reynolds number. Figure 3(a) indicates that at the low Reynolds number, the mean value of drag coefficient on the cross section decreases rapidly with increasing Reynolds number, and the mean value of drag coefficient on the cross section remains unchanged with increasing Reynolds number at the high Reynolds number. The simulation results in Figure 3(a) and (b) are in good agreement with the related research results. 


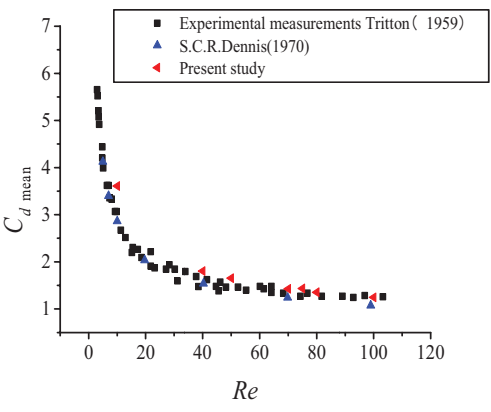

(a)

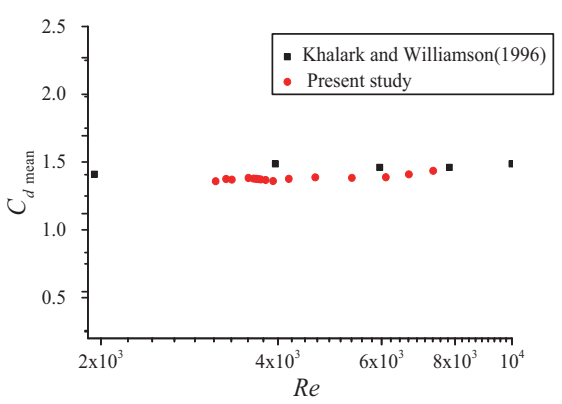

(b)

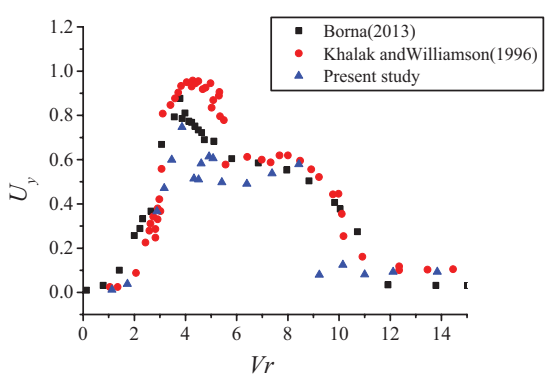

(c)

Figure 3 FSI of circular cylinder, the mass flow and damping ratio are 2.4 and 0.005417 , respectively. (a) Mean drag coefficient verses (low) Reynolds number, (b) Mean drag coefficient verses (high) Reynolds number and (c) Cross wind displacement verses reduced velocity, $3000 \leq \operatorname{Re} \leq 7500$.

In Figure 3(c), $U_{y}$ presents the dimensionless crosswind displacement, the expression of $U_{y}$ is defined as Equation (14).

$$
U_{y}=Y / D
$$

The simulation results in this paper are compared with those in the references (Borna, 2013; Khalak and Williamson, 1996). The above three groups of data appear the initial excitation branch, upper branch and lower branch in Figure 3(c). The simulation results agree well with the research results of references.

The aerodynamic coefficients of D-shape iced conductor varying with the reduced velocity under the condition of wind attack angle $0^{\circ}$ are shown in Figure 4. Where, "Rigid" represents the numerical simulation of flow around a rigid model; "1DOF" represents a one-degree-of-freedom fluid-structure interaction model with the consideration of the across-wind vibrating. 


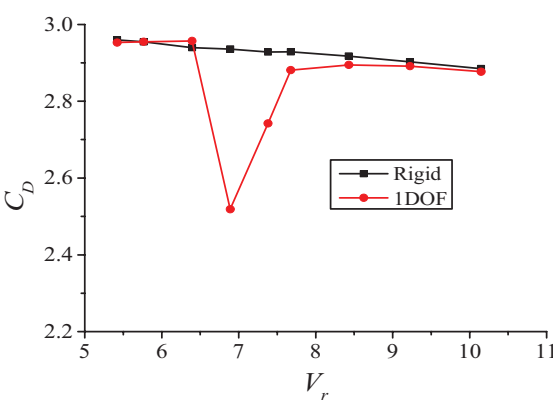

(a)

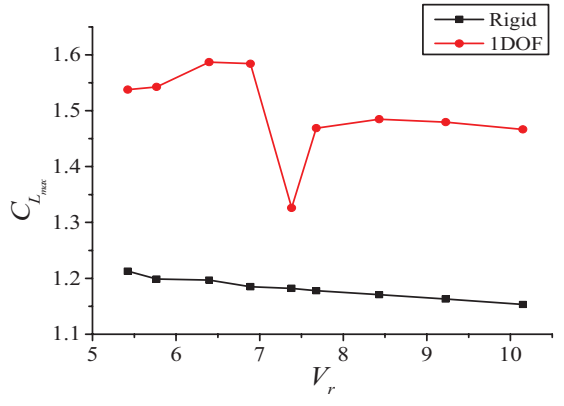

(b)

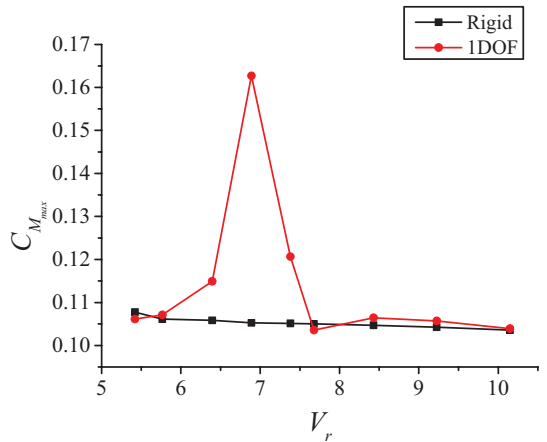

(c)

Figure 4 Variation of aerodynamic coefficients with reduced velocity. (a) Mean value of drag coefficient varying with $V_{r}$, (b) Maximum lift coefficient varying with $V_{r}$ and (c) Maximum moment coefficient varying with $V_{r}$.

It can be seen from Figure 4(a) that in 1DOF model, the mean value of drag coefficient is significantly lower than the rigid model at the lock in region, while the maximum lift coefficient is always greater than that of the Rigid model. It indicates that the across-wind vibration has a greater effect on the lift of 1DOF model. The maximum values of the moment coefficients calculated by the above two models are basically the same under various reduced velocity, however, the moment coefficient has a significant peak phenomenon at the reduced velocity equal to 6.89. In Figure 4, the sudden variation of aerodynamic coefficients at the reduced velocity of 6.89 is mainly due to vortex-induced vibration, which will be explained carefully in Figure 5.

Figure 5 shows the variation of frequency ratio, Strouhal number $\left(S_{t}\right)$ and displacement with reduced velocity. The frequency spectrum analysis from the 


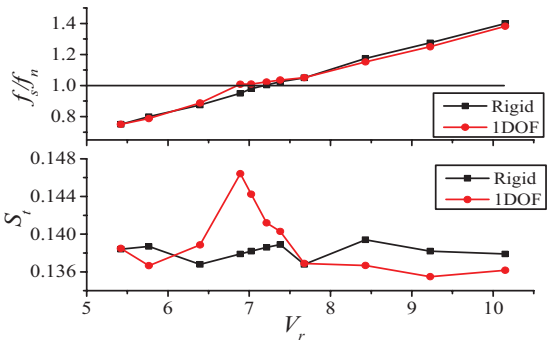

(a)

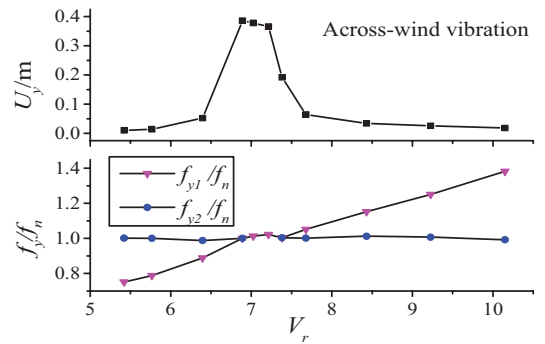

(b)

Figure 5 Spectrum analysis, displacement and St vs. reduced wind velocity. (a) Frequency and St varying with $V_{r}$ and (b) Displacement and frequency ratio varying with $V_{r}$.

time history curve of lift coefficient shows that there are two main frequencies in the unlocked region, one is about the natural frequency of the D-shape iced conductor $\left(f_{y 2}\right)$ and the other is about equal to the shedding frequency of the vortex $\left(f_{y 1}\right)$. These parameters are defined as follows:

$$
S_{t}=f_{y 1} D / V
$$

Where, the parameters $V$ and $D$ are consistent with the definitions above.

It can be seen from Figure 5(a) that the vortex shedding frequency of the 1DOF model is quite close to that of the Rigid model while $V_{r}<6.890$, and the ratio of $f_{s}$ to $f_{n}$ increases with $V_{r}$ linearly. In this reduced velocity range, the Strouhal number decreases first and then increases, reaching the maximum $\left(S_{t}=0.146\right)$ at $V_{r}=6.890$. When the $V_{r}$ belongs to the range of $6.890 \sim 7.677$, the vortex shedding frequency basically remains steady, which is similar to the "lock-in" phenomenon of vortex-induced vibration on circular cylinder. The frequency ratio is approximately equal to 1 , and the corresponding Strouhal number decreases. Meanwhile, the amplitude of the vibration displacement in across-wind direction also decreases in this lockin region. When $V_{r}>7.677$, the vortex shedding frequency escapes from the lock-in region, the frequency increases with the increase of the reduced velocity, the corresponding Strouhal number and crosswind displacement basically remains steady; in addition, the Strouhal number of 1DOF model is less than that of the rigid model.

It can be seen from Figure 5(b) that the dimensionless vibration displacement increases when $V_{r}>6.395$ and reaches its maximum value at $V_{r}=6.890$. The corresponding amplitude decreases rapidly when the reduced velocity breaks away from the lock-in region. From the frequency spectrum analysis of the time history curve of vibration displacement, it can be found 
that there are two kinds of frequencies existed outside the lock-in region, one is the vortex shedding frequency, and the other is the natural frequency of cylinder while there is only one dominant frequency in the lock-in region.

\subsection{Influence of Multi-Degree-of-Freedom on Vibration}

The setting of the simulation parameters is the same as that of the 1DOF model. Here, the other two fluid-structure interaction models are also simulated. A 2DOF model which includes along-wind and across-wind vibrating and a 3DOF model which includes along-wind, across-wind and torsional vibrating. Since the main purpose of this paper here is to investigate the vortex-induced vibration of $\mathrm{D}$-shaped iced conductor, it is also significant to study the vortex shedding pattern and vortex street of the D-shaped iced conductor at different reduced velocities. The flow field distribution of vortex shedding pattern and vortex street of the 2DOF model are presented in Appendix. It revealed that the vortex separation points of the D-shaped iced cross section appear at the upper and lower corners. The vortexes at the wake region are relatively simple, and they are alternately arranged in two rows. In the corresponding wake region, the vortex structure mainly presents the " $2 \mathrm{P}$ " vortex pattern. From the appendix it seems that the intensity of the vortices at $V_{r}=6.890$ is the highest in compare to the other two vortices at $V_{r}=5.766$ and $V_{r}=9.266$.

Figure 6 shows the characteristic values calculated by the above three kinds of fluid-structure interaction models varying with reduced velocity.

It can be seen from Figure 6 that the simulation values obtained by the 2DOF model are basically consistent with those of the 1DOF. The reduced velocity corresponding to the maximum characteristic values calculated by the above three models is the same one. With the torsion degree of freedom being taken into account, the characteristic values (except the mean and standard deviation values of drag coefficient) vary greatly, especially in the lock-in region, the values is significantly larger than that of the other two cases. The above analysis shows that the effect of torsion degree of freedom on across-wind vibrating is obvious while the effect of along-wind degree of freedom on across-wind vibrating is not obvious. In addition, as can be seen from the appendix, when the reduced velocity is 6.89 , the high-intensity vortices can also be used to explain the reasons why the lift coefficient and the dimensionless crosswind displacement reaches its maximum value in Figure 6.

Figure 7 shows the variation of the vortex shedding frequency ratio and the Strouhal number with reduced velocity for the three kinds of fluid-structure interaction models. 


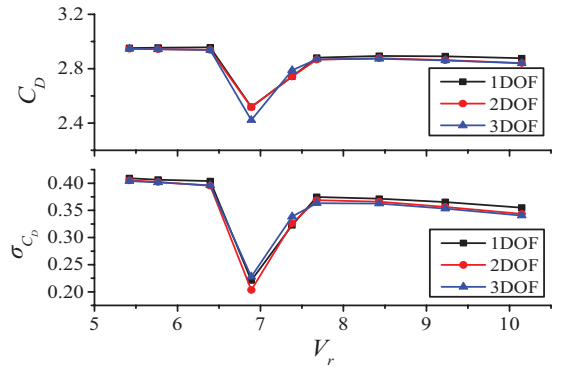

(a)
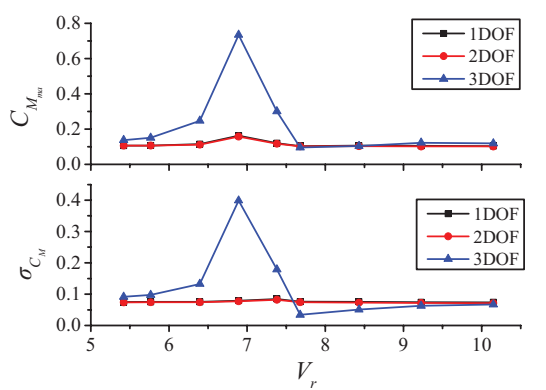

(c)

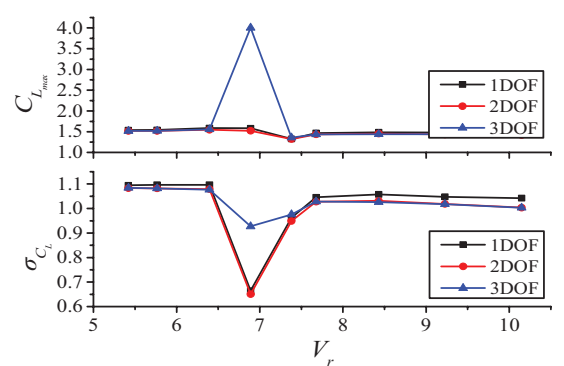

(b)
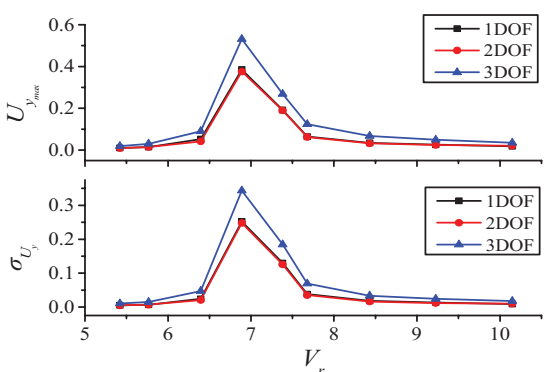

(d)

Figure 6 The comparison of fluid-structure interaction vibration under different DOF. (a) Mean and standard deviation values of drag coefficient varying with $V_{r}$, (b) Maximum lift coefficient and its standard deviation values varying with $V_{r}$, (c) Maximum moment coefficient and its standard deviation values varying with $V_{r}$ and (d) Displacement and its standard deviation values varying with $V_{r}$.

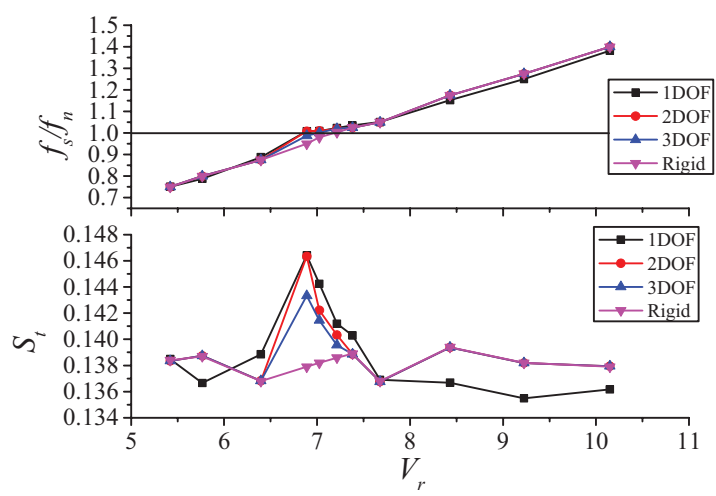

Figure 7 Variation of frequency ratio and St with reduced velocity. 
With the consideration of the other two degrees of freedom, the vortex shedding frequency is slightly higher than that of the 1DOF model, which indicates that the degree of freedom will increase the velocity of vortex shedding. In addition, when the reduced velocity reaches 6.890 , the above three kinds of models show the lock-in phenomenon. When $V_{r}<6.890$, the Strouhal number tends to increase with the increase of $V_{r}$, and reaches the maximum value at $V_{r}=6.890$. Then the Strouhal number decreases with the increase of $V_{r}$, and finally almost remains steady. When $V_{r}>7.677$, the Strouhal number of 2DOF is consistent with that of the 3DOF model, and larger than that of the 1DOF model.

The above analysis show that compared with the 1DOF and 2DOF models, the 3DOF model has various influences on the aerodynamic characteristics, vibration displacement, and the vortex shedding frequency of the D-shape iced conductor. When the lock-in phenomenon occurs, the drag coefficient of the 3DOF model is reduced, while the lift coefficient, moment coefficient and vibration displacement of the iced conductor are increased.

Figure 8 shows the along-wind, torsional displacement and their standard deviation values varying with reduced velocity. The definition of the dimensionless torsional displacement is consistent with that of crosswind (See the formula (14) for details).

As shown in Figure 8(a): the maximum displacement in along-wind direction and its standard deviation values of the 3DOF model is larger than that of the 2DOF model under different velocities. It can be seen from Figure 8(b) that the variation law of torsional displacement with reduced

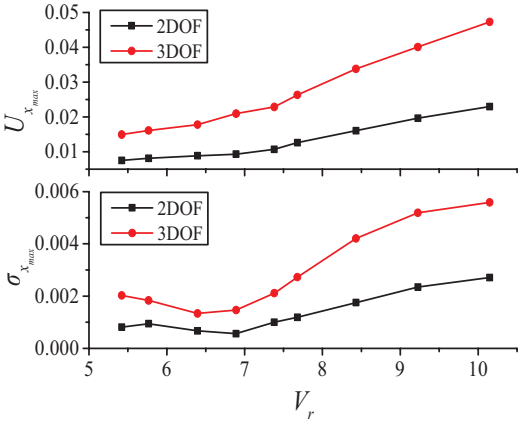

(a)

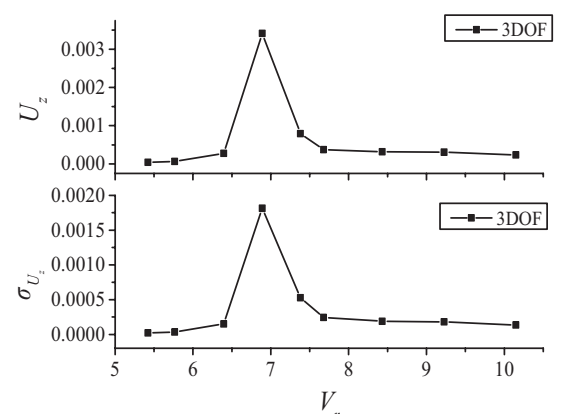

(b)

Figure 8 Vibration displacement varying with $V_{r}$ ((a) Maximum vibration displacement in along-wind direction and its standard deviation values varying with $V_{r}$ and (b) Torsional displacement and its standard deviation values varying with $V_{r}$ ). 
velocity is similar to the variation of Strouhal number with reduced velocity. In general, compared to the vibration displacement of the along-wind and torsional direction, the displacement in the cross flow direction is the highest.

\subsection{Influence of Wind Attack Angle}

The cross section of iced conductor tends to be non-circular, which leads to the aerodynamic coefficients varying with various wind attack angles. Therefore, the initial wind attack angle of $10^{\circ}, 20^{\circ}$ and $30^{\circ}$ are discussed in the 3DOF model.

\subsubsection{Aerodynamic forces under different initial wind attack angles}

Figure 9 shows the aerodynamic coefficient varying with the reduced velocity under different initial wind attack angles. Since the cross section of the iced

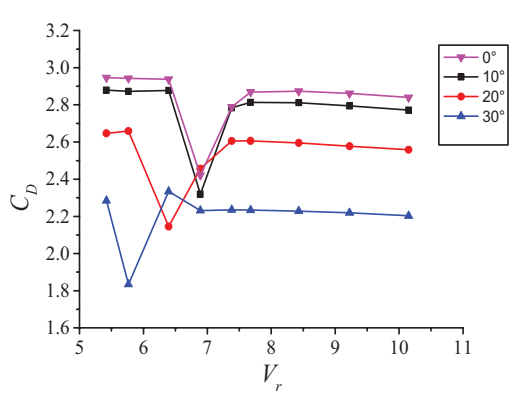

(a)

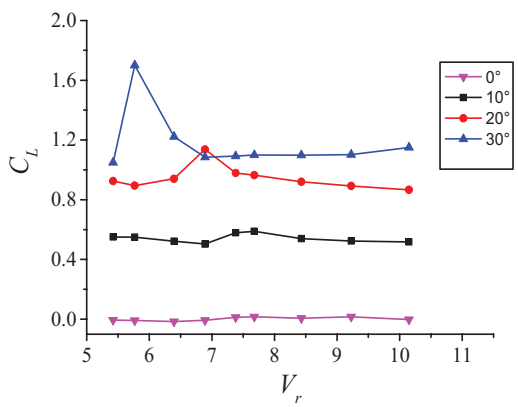

(b)

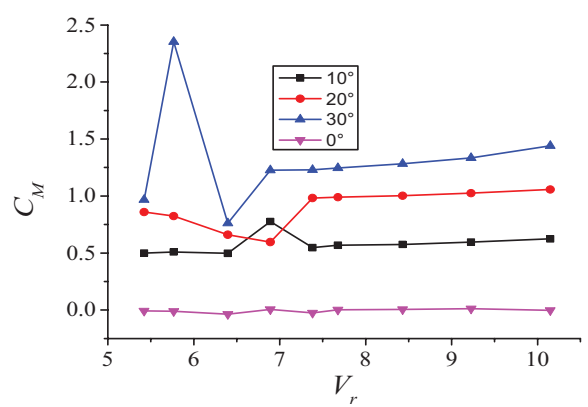

(c)

Figure 9 Aerodynamic force coefficients varying with $V_{r}$ under different initial attack angles. (a) Mean value of $C_{D}$ varying with $V_{r}$, (b) Mean value of $C_{L}$ varying with $V_{r}$ and (c) Mean value of $C_{M}$ varying with $V_{r}$. 
conductor is no longer regular, the mean value of the aerodynamic coefficient is more meaningful than the maximum value.

It also can be seen from Figure 9(a) that when the reduced velocity is the same, the larger the wind attack angle, the smaller the corresponding mean value of drag coefficient. This is mainly due to the larger wind attack angle corresponds to the smaller windward area. In addition, the mean value of lift and moment coefficient almost increases with the increase of wind attack angle. When the wind attack angle is not equal to $0^{\circ}$, the aerodynamic coefficients vary drastically at a certain reduced velocity, especially, the variation of mean value of drag coefficient with various wind attack angles. In addition, when wind attack angle increases, the reduced wind velocity of "lock-in" phenomenon also decreases.

\subsubsection{Displacement under different initial wind attack angles}

Figure 10 shows the dimensionless displacement varying with reduced velocity under different initial wind attack angles in the 3DOF model.

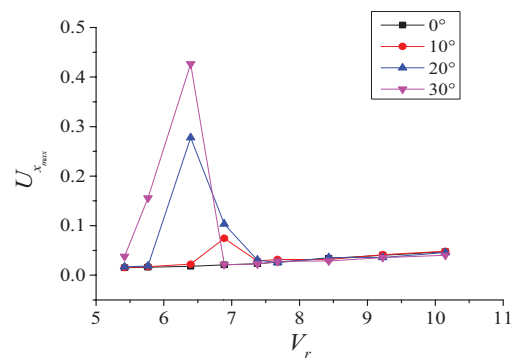

(a)

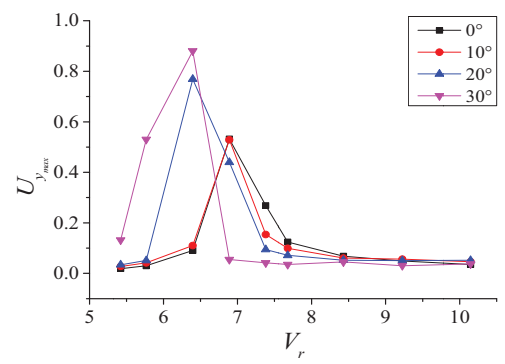

(b)

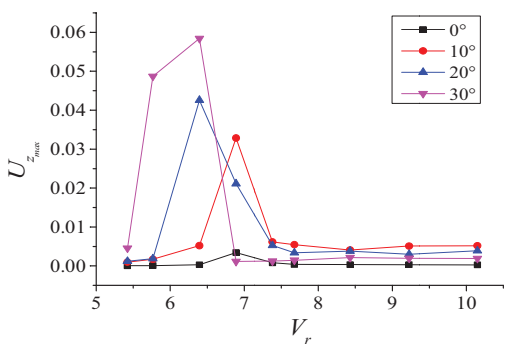

(c)

Figure 10 Vibration displacement under different initial attack angles in the 3DOF model. (a) Windward displacement, (b) Crosswind displacement and (c) Torsional displacement. 
The aerodynamic force on the iced conductor varies with the wind attack angle. The variation of aerodynamic force eventually leads to different structural displacement response. Compared with the data from Figure 10, it is found that the maximum vibration displacement of the conductor increases with the increase of the wind attack angle. In addition, with the increase of wind attack angle, the reduced velocity corresponding to the maximum vibration displacement becomes smaller, which shows that the influence of the initial wind attack angle on the vibration displacement of the conductor is obvious. It is also worth noting that the reduced velocity corresponding to the maximum vibration displacement is not exactly the same as that corresponding to the maximum aerodynamic coefficient.

\section{Conclusion}

This paper presents a numerical study of three kinds of fluid-structure interaction models for D-shape conductor using the FLUENT software. The effects of degree of freedom, reduced velocity and wind attack angle on the aerodynamic response of the iced conductor are discussed. The main conclusions are as follows:

1. The mean value of drag coefficient of the $1 \mathrm{DOF}$ model is slightly less than that of the rigid model except for that it will dropped sharply at the reduced velocity of $V_{r}=7$. The maximum value of lift coefficient is always larger than that of rigid model, and the maximum value of torsion coefficient is basically close to the value of rigid model except for the reduced velocity in the range of $6.0 \sim 7.677$.

2. The rotational freedom has certain influence on the vibration in acrosswind direction. However, the effect of along-wind degree of freedom on across-wind vibrating is not obvious.

3. The mean value of drag coefficient decreases with the increase of wind attack angle, while the lift and moment coefficient increase with the increase of wind attack angle.

4. With the increase of the wind attack angle, the vibration displacement of the iced conductor increases obviously in the lock-in region, and the reduced velocity corresponding to the maximum displacement becomes smaller. When the maximum amplitude of vibration displacement occurs, the corresponding reduced velocity is not entirely consistent with that of the maximum aerodynamic force. 
164 Y. Yi et al.

\section{Acknowledgments}

The work described in this paper was fully supported by the National Natural Science Foundation of China (Grant No.: 51478069 and 51778097) and the Chongqing Science \& Technology Commission (No. cstc2017jcyjJB0210, cstc2018jscx-msybX0284).

\section{Appendix}

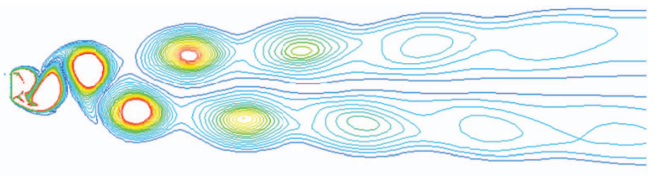

(a)

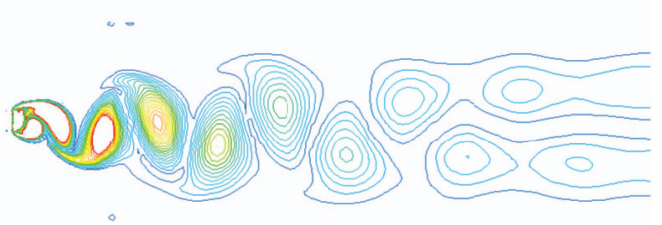

(b)

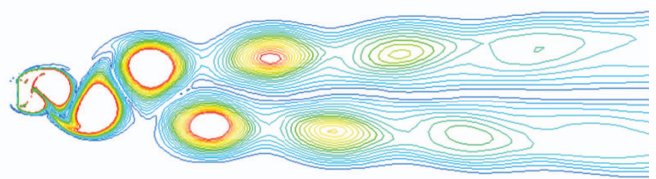

(c)

Figure A1 Vortex structure of 2DOF model varies with different $V_{r}$. (a) $V_{r}=5.766$, (b) $V_{r}=6.890$ and (c) $V_{r}=9.226$.

\section{References}

Al-jamal, H. and Dalton, C. (2004). Vortex induced vibrations using Large Eddy Simulation at a moderate Reynolds number. Journal of Fluids and Structures, 19, 73-92. 
Arun, K. S. (2013). Unsteady flow past a finite square cylinder mounted on a wall at low Reynolds number. Computers and Fluids, 88, 599-615.

Borna, A. (2014). Prediction of galloping of transmission line conductors by a computational aeroelastic approach. Doctoral dissertation.

Borna, A., Habashi, W. G., Nadarajah, S. K. and McClure, G. (2011). A computational aeroelastic approach to predict galloping of iced conductors with three degrees of freedom. In Proc. 14th Int. Workshop Atmospher. Icing Struct, 137-144.

Borna, A., Habashi, W. G., Mcclure, G., et al. (2013). CFD-FSI simulation of vortex-induced vibrations of a circular cylinder with low mass-damping. Wind and Structures, 16, 411-431.

Chabart, O. and Lilien, J. L. (1998). Galloping of electrical Iines in wind tunnel facilities. Journal of wind Engineering and Industrial Aerodynamics, 74, 967-976.

Dennis, S. C. R. and Chang, G. Z. (1970). Numerical solutions for steady flow past a circular cylinder at Reynolds numbers up to 100. Journal of Fluid Mechanics, 42, 471-489.

Dyke, P. V. and Laneville, A. (2008). Galloping of a single conductor covered with a D-section on a high-voltage overhead test line. Journal of Wind Engineering and Industrial Aerodynamics, 96, 1141-1151.

Gabbai, R. D. and Benaroya, H. (2005). An overview of modeling and experiments of vortex-induced vibration of circular cylinders. Journal of Sound and Vibration, 282, 575-616.

Govardhan, R. and Williamson, C. H. K. (2000). Modes of vortex formation and frequency response for a freely vibrating cylinder. Journal of Fluid Mechanics, 420, 85-130.

Griffin, O. M. and Koopmarm, G. H. (1977). The vortex-excited lift and reaction forces on resonantly vibrating cylinders. Journal of Sound and Vibration, 54, 435-448.

Griffin, O. M. and Koopmarm, G. H. (1980). Vortex-excited cross-flow vibrations of a single cylindrical tube. ASME, Journal of Pressure Vessel Technology, 102, 158-166.

Guilmineau, E. and Queutey, P. (2004). Numerical simulation of vortexinduced vibration of a circular cylinder with low mass-damping in a turbulent flow. Journal of Fluids and Structures, 19, 449-466.

Gurung, C. B. Yamaguchi, H. and Yukino, T. (2002). Identification of large amplitude wind-induced vibration of ice-accreted transmission lines based on field observed data. Engineering Structures, 24, 179-188. 
Kamakoti, R. and Shyy, W. (2004). Fluid-structure interaction for aeroelastic applications. Progress in Aerospace Sciences, 40, 535-558.

Khalak, A. and Williamson, C. H. K. (1996). Dynamics of a hydroelastic cylinder with very low mass and damping. Journal of Fluids and Structures, 10, 455-472.

Li, X. M., Zhu, K. J. and Liu, B. (2013a). Experimental simulation on aerodynamic character of D-shaped iced conductor. In Advanced Materials Research, 614, 1405-1409.

Li, X. M., Zhu, K. J. and Liu, B. (2013b). Numerical and experimental simulation on aerodynamic character of crescent-shaped iced conductor. Applied Mechanics and Materials, 275, 622-627.

Liu, B., Zhu, K. J., Li, X. M. and Zhan, X. P. (2013). Hysteresis phenomenon in the galloping of the D-shape iced conductor. Mathematical Problems in Engineering, 2013, 1-11.

Liu, Y., Tang, A. P., Liu, K. T. and Tu, J. W. (2015). Numerical simulation on the aerodynamic force of the iced conductor for different angles of attack. Engineering Review, 35, 157-169.

Lou, W., Lv, J., Huang, M. F., Yang, L. and Yan, D. (2014). Aerodynamic force characteristics and galloping analysis of iced bundled conductors. Wind and Structures, 18, 135-154.

Ma, W. Y., Li, X. N. and Gu, M. (2014). Experimental investigation and theoretical analysis on galloping of iced conductors. Advanced Materials Research, 860, 1551-1558.

Pan, Z. Y., Cui, W. C. and Miao, Q. M. (2007). Numerical simulation of vortex-induced vibration of a circular cylinder at low mass-damping using RANS code. Journal of Fluids and Structure, 23-37.

Sarpkaya, T. (2004). A critical review of the intrinsic nature of vortex-induced vibrations. Journal of Fluids and Structures, 19, 389-447.

Shimizu, M., Ishihara, T. and Phue, P. V. (2004). A wind tunnel study on steady and unsteady aerodynamic characteristics of ice-accreted transmission lines. Proc. of the 18th Symposium on Wind Engineering, Tokyo (Japan), 245-250.

Tritton, D. J. (1959). Experiments on the flow past a circular cylinder at low Reynolds numbers [J]. Journal of Fluid Mechanics Digital Archive, 6(04), 21.

Wang, Z. J. and Zhou, Y. (2005). Vortex-induced vibration characteristic of an elastic square cylinder on fixed supports. Journal of Fluids and Structures, 127, 241-249. 
Williamson, C. H. K. and Govardhan, R. (2004). Vortex-induced vibrations. Annual Review of Fluid Mechanics, 36, 413-455.

Williamson, C. H. K. and Govardhan, R. (2008). A brief review of recent results in vortex-induced vibrations. Journal of Wind Engineering and Industrial Aerodynamics, 96, 713-735.

Yan, Z., Li, Z., Savory, E. and Lin, W. E. (2013). Galloping of a single iced conductor based on curved-beam theory. Journal of Wind Engineering and Industrial Aerodynamics, 123, 77-87.

Yang, J., Preidikman, S. and Balaras, E. (2008). A strongly coupled, embedded-boundary method for fluid-structure interactions of elastically mounted rigid bodies. Journal of Fluids and Structures, 24, 167-182.

Yu, P., Popplewell, N. and Shah, Ah. (1995). Instability trends of inertially coupled galloping: Part I Initiation. Journal of Sound and Vibration, 183, 663-678.

Zhang, D., He, Z., Huang, Z. and Jiang, W. (2017). Isogeometric collocation method for the galloping of an iced conductor. Journal of Engineering Mechanics, 143, 04017009.

\section{Biographies}

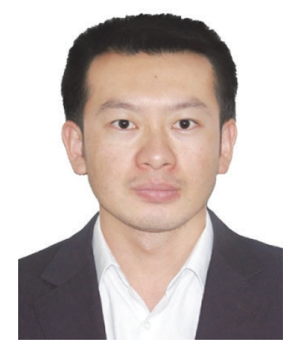

You Yi is a Ph.D. student at the Chongqing University since 2016. He attended the Harbin Engineering University where he received his B.Sc. in Ship and Ocean Engineering in 2010. He then received his M.Sc. in Solid Mechanics from the Beijing University of Science and Technology in 2014. His Ph.D. work centers on structural wind engineering. 


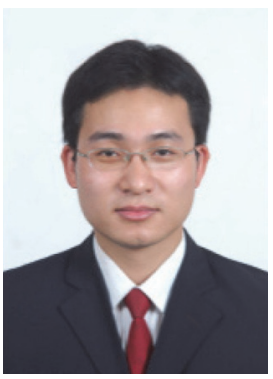

Yan Zhitao received his B.Sc degree in Civil Engineering from Nanchang University and received his M.Sc. and Ph.D. degree in Civil Engineering from Chongqing University. Now, he is a professor at Chongqing University and Chongqing University of Science and Technology. His main research fields are structural wind engineering and computational fluid dynamics.

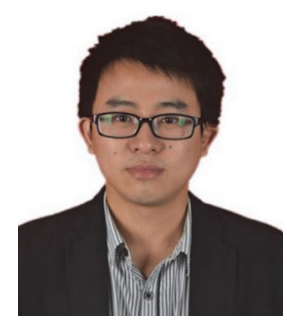

Nie Xiaochun received his B.Sc degree in Engineering Management from Southwest University of Science and Technology, and then received his M.Sc. degree in Civil Engineering from Chongqing University. Now, He is a Ph.D. student at the Chongqing University since 2016. His main research fields are structural wind engineering and nonlinear vibration. 


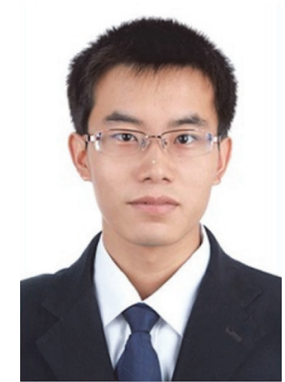

Yang Xiaogang is a Ph.D. student at the Chongqing University since 2015. He received his B.Sc degree in Civil Engineering from Chongqing University. His main research field is computational fluid dynamics.

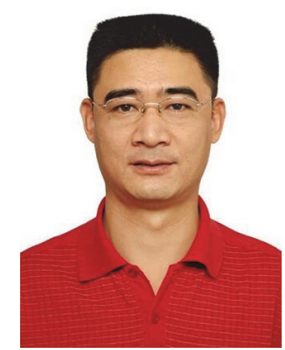

Li Wensheng received his B.Sc degree in Metal Material from Wuhan University of Water Resources and Hydropower. Now, He is a senior engineer in Electric Power Research Institute, State Grid Xinjiang Electric Power Company. 


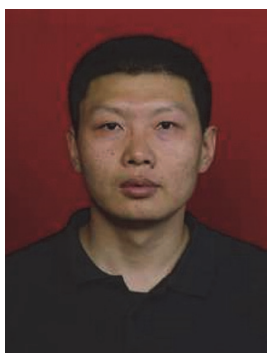

He Cheng received his B.Sc degree in Metal Material from Wuhan University of Water Resources and Hydropower. Now, He is also a senior engineer in Electric Power Research Institute, State Grid Xinjiang Electric Power Company.

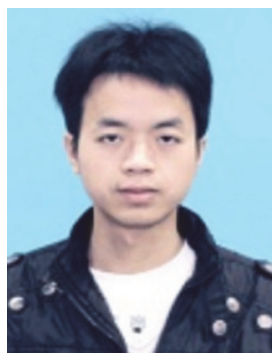

Liu Jun is a postgraduate student of School of Civil Engineering of Chongqing University. He received his B.Sc degree from Northwestern Polytechnical University. His work centers on computational fluid dynamics. 